\title{
pLoc_Deep-mGpos: Predict Subcellular Localization of Gram Positive Bacteria Proteins by Deep Learning
}

\author{
Zhe Lu' ${ }^{1}$, Kuo-Chen Chou ${ }^{1,2}$ \\ ${ }^{1}$ Computer Science, Jingdezhen Ceramic Institute, Jingdezhen, China; ${ }^{2}$ Gordon Life Science Institute, Boston, MA, \\ USA
}

Correspondence to: Zhe Lu，454170054@qq.com; Kuo-Chen Chou，kcchou@gordonlifescience.org,

kcchou38@gmail.com

Keywords: Pandemic Coronavirus, Multi-Label System, Gram Positive Proteins, Learning at Deeper Level, Five-Steps Rule, PseAAC

Received: April 8, 2020 Accepted: May 8, $2020 \quad$ Published: May 11, 2020

Copyright $\odot 2020$ by author(s) and Scientific Research Publishing Inc.

This work is licensed under the Creative Commons Attribution International License (CC BY 4.0).

http://creativecommons.org/licenses/by/4.0/

\section{(c) (i) Open Access}

\section{ABSTRACT}

The recent worldwide spreading of pneumonia-causing virus, such as Coronavirus, COVID-19, and H1N1, has been endangering the life of human beings all around the world. In order to really understand the biological process within a cell level and provide useful clues to develop antiviral drugs, information of Gram positive bacteria protein subcellular localization is vitally important. In view of this, a CNN based protein subcellular localization predictor called "pLoc_Deep-mGpos" was developed. The predictor is particularly useful in dealing with the multi-sites systems in which some proteins may simultaneously occur in two or more different organelles that are the current focus of pharmaceutical industry. The global absolute true rate achieved by the new predictor is over $99 \%$ and its local accuracy is around $92 \%-99 \%$. Both are transcending other existing state-of-the-art predictors significantly. To maximize the convenience for most experimental scientists, a user-friendly web-server for the new predictor has been established at http://www.jci-bioinfo.cn/pLoc_Deep-mGpos/, which will become a very powerful tool for developing effective drugs to fight pandemic coronavirus and save the mankind of this planet.

\section{INTRODUCTION}

Knowledge of the subcellular localization of proteins is crucially important for fulfilling the following two important goals: 1) revealing the intricate pathways that regulate biological processes at the cellular level [1,2].2) selecting the right targets [3] for developing new drugs.

With the avalanche of protein sequences in the post-genomic age, we are challenged to develop com- 
putational tools for effectively identifying their subcellular localization purely based on the sequence information.

In 2019, a very powerful predictor, called "pLoc_bal-mGpos" [4], was developed for predicting the subcellular localization of Gram positive proteins based on their sequences information alone. It has the following remarkable advantages. 1) Most existing protein subcellular location prediction methods were developed based on the single-label system in which it was assumed that each constituent protein had one, and only one, subcellular location (see, e.g., [5-7] and a long list of references cited in a review papers [8]). With more experimental data uncovered, however, the localization of proteins in a cell is actually a multi-label system, where some proteins may simultaneously occur in two or more different location sites. This kind of multiplex proteins often bears some exceptional functions worthy of our special notice [2]. And the pLoc_bal-mGpos predictor [4] can cover this kind of important information missed by most other methods since it was established based on the multi-label benchmark dataset and theory. 2) Although there are a few methods (see, e.g., $[9,10]$ ) that can be used to deal with multi-label subcellular localization for proteins, the prediction quality achieved by pLoc_bal-mGpos [4] is overwhelmingly higher, particularly in the absolute true rate. 3) Although the pLoc_bal-mGpos predictor [4] has the aforementioned merits, it has not been trained at a deeper level yet [11-14].

The present study was initiated in an attempt to address this problem. As done in pLoc_bal-mGpos [4] as well as many other recent publications in developing new prediction methods (see, e.g., [15, 16], the guidelines of the 5-step rule [17] are followed. They are about the detailed procedures for 1) benchmark dataset, 2) sample formulation, 3) operation engine or algorithm, 4) cross-validation, and 5) web-server. But here our attentions are focused on the procedures that significantly differ from those in developing the predictor pLoc_bal-mGpos [4].

\section{MATERIALS AND METHODS}

\subsection{Benchmark Dataset}

The benchmark dataset used in this study is exactly the same as that in pLoc_bal-mGpos [4]; i.e.,

$$
\mathbb{S}=\mathbb{S}_{1} \cup \mathbb{S}_{2} \cup \mathbb{S}_{3} \cup \mathbb{S}_{4}
$$

where $\mathbb{S}_{1}$ only contains the Gram-positive bacterial protein samples from the "Cell membrane" organelle (cf. Table 1), $\mathbb{S}_{2}$ only contains those from the "Cell wall", and so forth; $U$ denotes the symbol for "union" in the set theory. For readers' convenience, their detailed sequences and accession numbers (or ID codes) are given in Supporting Information S1 that is also available at

http://www.jci-bioinfo.cn/pLoc_bal-mGpos/Supp1.pdf in which none of proteins included has $\geq 25 \%$ sequence identity to any other in the same subset (subcellular location).

\subsection{Proteins Sample Formulation}

Now let us consider the $2^{\text {nd }}$ step of the 5-step rule [17]; i.e., how to formulate the biological sequence

Table 1. Comparison with the state-of-the-art method in predicting Gram positive protein subcellular localization ${ }^{\mathrm{a}}$.

\begin{tabular}{cccccc}
\hline Predictor & Aiming $(\uparrow)^{\mathrm{a}}$ & ${\text { Coverage }(\uparrow)^{\mathrm{a}}}$ & Accuracy $(\uparrow)^{\mathrm{a}}$ & Absolute true $(\uparrow)^{\mathrm{a}}$ & Absolute false $(\downarrow)^{\mathrm{a}}$ \\
\hline pLoc_bal-mGpos $^{\mathrm{b}}$ & $98.31 \%$ & $98.31 \%$ & $98.28 \%$ & $98.28 \%$ & $0.00 \%$ \\
pLoc_Deep-mGpos $^{\mathrm{c}}$ & $99.24 \%$ & $99.24 \%$ & $99.24 \%$ & $99.24 \%$ & $0.00 \%$ \\
\hline
\end{tabular}

${ }^{a}$ See Equation (4) for the definition of the metrics. ${ }^{b}$ See [4], where the reported metrics rates were obtained by the jackknife test on the benchmark dataset of Supporting Information S1 that contains experiment-confirmed proteins only. ${ }^{\circ}$ The proposed predictor; to assure that the test was performed on exactly the same experimental data as reported in [4] for pLoc_bal-mGpos. 
samples with an effective mathematical expression that can truly reflect their essential correlation with the target concerned. Given a protein sequence $P$, its most straightforward expression is

$$
\mathbf{P}=\mathrm{R}_{1} \mathrm{R}_{2} \mathrm{R}_{3} \mathrm{R}_{4} \mathrm{R}_{5} \mathrm{R}_{6} \mathrm{R}_{7} \cdots \mathrm{R}_{L}
$$

where $L$ denotes the protein's length or the number of its constituent amino acid residues, $\mathrm{R}_{1}$ is the $1^{\text {st }}$ residue, $R_{2}$ the $2^{\text {nd }}$ residue, $R_{3}$ the $3^{\text {rd }}$ residue, and so forth. Since all the existing machine-learning algorithms\} can only handle vectors as elaborated in [3], one has to convert a protein sample from its sequential expression (Equation (2)) to a vector. But a vector defined in a discrete model might completely miss all the sequence-order or pattern information. To deal with this problem, the Pseudo Amino Acid Composition [18] or PseAAC [19]. Ever since then, the concept of "Pseudo Amino Acid Composition" has been widely used in nearly all the areas of computational proteomics with the aim to grasp various different sequence patterns that are essential to the targets investigated (see, e.g., [20-30] as well as a long list of references cited in [31]). Because it has been widely and increasingly used, recently three powerful open access soft-wares, called "PseAAC-Builder" [32], "propy" [33], and "PseAAC-General" [34], were established: the former two are for generating various modes of special PseAAC [35]; while the 3rd one for those of general PseAAC [17], including not only all the special modes of feature vectors for proteins but also the higher level feature vectors such as "Functional Domain" mode, "Gene Ontology" mode, and "Sequential Evolution" or "PSSM" mode. Encouraged by the successes of using PseAAC to deal with protein/peptide sequences, its idea and approach were extended to PseKNC (Pseudo K-tuple Nucleotide Composition) to generate various feature vectors for DNA/RNA sequences [36] that have proved

According to the concept of general PseAAC [17], any protein sequence can be formulated as a PseAAC vector given by very successful as well (see, e.g., [37].

$$
\mathbf{P}=\left[\begin{array}{llllll}
\Psi_{1} & \Psi_{2} & \cdots & \Psi_{u} & \cdots & \Psi_{\Omega}
\end{array}\right]^{\mathbf{T}}
$$

where $\mathrm{T}$ is a transpose operator, while the integer $\Omega$ is a parameter and its value as well as the components $\Psi_{u}(u=1,2, \cdots, \Omega)$ will depend on how to extract the desired information from the amino acid sequence of $\mathbf{P}$, as elaborated in [38]. Thus, by following exactly the same procedures as described in the Section 2.2 of [38], each of the protein samples in the benchmark dataset can be uniquely defined as a 8-D numerical vector as given in Supporting Information S2, which can also be directly downloaded at http://www.jci-bioinfo.cn/pLoc_bal-mGneg/Supp2.pdf.

\subsection{Installing Deep-Learning for Three Deeper Levels}

In this study, we use multilayer perceptron neural network model, which consists of 3 fully connected layers and was used to predict subcellular localization of multi-label Gram positive proteins, as illustrated in Figure 1. We set input layer with 14 neural unGranits which correspond to 14 features. Too many hidden layers would make network complexity bigger and suffer from the vanishing gradient problem while a model is constructed. Here, only two hidden layer is included. The hidden layer 1 is set as 200 neural units. The activation function is set as "relu". The second hidden layer has 100 neural units. The activation function is set the same as the hidden layer 1 . We end the model with 14 neural units and Sigmoid activation. To go with it, we use the binary_crossentropy loss and the adam (adaptive moment estimation) optimizer to train the model. The metrics is set as "accuracy". The batch size is set as 28 , and the epochs is 100 . The predicted results were decided by the output of the threshold $\theta$. If the output is greater than 0.5 , the outcome was true; otherwise, false. For more information about this, see [2], where the details have been clearly elaborated and hence there is no need to repeat here. For more information about this, see [11], where the details have been clearly elaborated and hence there is no need to repeat here.

The new predictor developed via the above procedures is called "pLoc_Deep-mGpos", where "pLoc_Deep" stands for "predict subcellular localization by deep learning", and "mGpos" for "multi-label Gram positive proteins". 


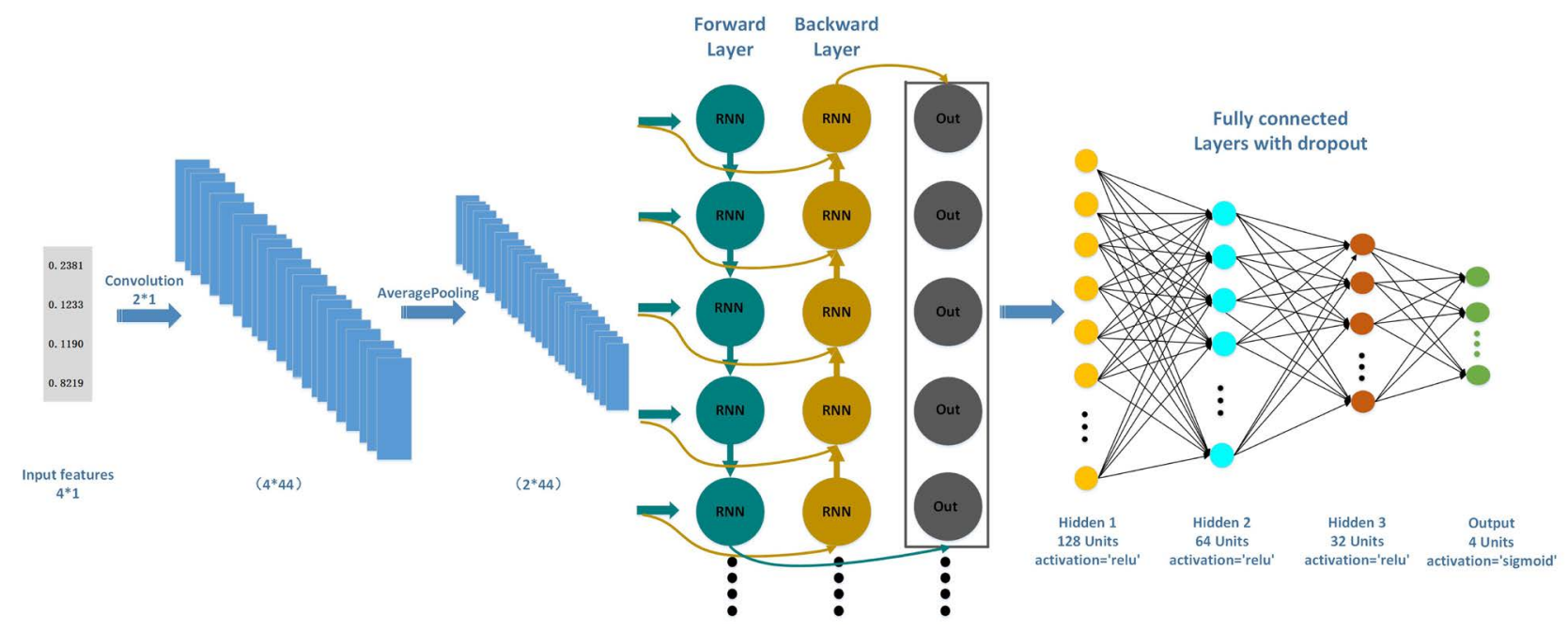

Figure 1. An illustration to show a dense neural network with 3 fully connected layers. Adapted from [11] with permission.

\section{RESULTS AND DISCUSSION}

According to the 5-step rules [17], one of the important procedures in developing a new predictor is how to properly evaluate its anticipated accuracy. To deal with that, two issues need to be considered. 1) What metrics should be used to quantitatively reflect the predictor's quality? 2) What test method should be applied to score the metrics?

\subsection{A Set of Five Metrics for Multi-Label Systems}

Different from the metrics used to measure the prediction quality of single-label systems, the metrics for the multi-label systems are much more complicated [39]. To make them more intuitive and easier to understand for most experimental scientists, here we use the following intuitive Chou's five metrics [40] or the "global metrics" that have recently been widely used for studying various multi-label systems (see, e.g., $[41,42])$. For the current study, the set of global metrics can be formulated as:

$$
\left\{\begin{array}{l}
\text { Aiming } \uparrow=\frac{1}{N^{\mathrm{q}}} \sum_{k=1}^{N^{\mathrm{q}}}\left(\frac{\left\|\mathbb{L}_{k} \cap \mathbb{L}_{k}^{*}\right\|}{\left\|\mathbb{L}_{k}^{*}\right\|}\right),[0,1] \\
\text { Coverage } \uparrow=\frac{1}{N^{\mathrm{q}}} \sum_{k=1}^{N^{\mathrm{q}}}\left(\frac{\left.\left\|\mathbb{L}_{k} \cap \mathbb{L}_{k}^{*}\right\|\right)}{\left\|\mathbb{L}_{k}\right\|}\right),[0,1] \\
\text { Accuracy } \uparrow=\frac{1}{N^{\mathrm{q}}} \sum_{k=1}^{N^{\mathrm{q}}}\left(\frac{\left.\left\|\mathbb{L}_{k} \cap \mathbb{L}_{k}^{*}\right\|\right)}{\left\|\mathbb{L}_{k} \cup \mathbb{L}_{k}^{*}\right\|}\right),[0,1] \\
\text { Absolute true } \uparrow=\frac{1}{N^{\mathrm{q}}} \sum_{k=1}^{N^{\mathrm{q}}} \Delta\left(\mathbb{L}_{k}, \mathbb{L}_{k}^{*}\right),[0,1] \\
\text { Absolute false } \downarrow=\frac{1}{N^{\mathrm{q}}} \sum_{k=1}^{N^{\mathrm{q}}}\left(\frac{\left.\left\|\mathbb{L}_{k} \cup \mathbb{L}_{k}^{*}\right\|-\left\|\mathbb{L}_{k} \cap \mathbb{L}_{k}^{*}\right\|\right),[1,0]}{M}\right)
\end{array}\right.
$$

where $N^{\mathrm{q}}$ is the total number of query proteins or tested proteins, $M$ is the total number of different labels for the investigated system (for the current study it is $L_{\text {cell }}=4$ ), \|\| means the operator acting on 
the set therein to count the number of its elements, $U$ means the symbol for the "union" in the set theory, $\cap$ denotes the symbol for the "intersection", $\mathbb{L}_{k}$ denotes the subset that contains all the labels observed by experiments for the $k$-th tested sample, $\mathbb{L}_{k}^{*}$ represents the subset that contains all the labels predicted for the $k$-th sample, and

$$
\Delta\left(\mathbb{L}_{k}, \mathbb{L}_{k}^{*}\right)=\left\{\begin{array}{l}
1, \text { if all the labels in } \mathbb{L}_{k}^{*} \text { are identical to those in } \mathbb{L}_{k} \\
0, \text { otherwise }
\end{array}\right.
$$

In Equation (4), the first four metrics with an upper arrow $\uparrow$ are called positive metrics, meaning that the larger the rate is the better the prediction quality will be; the $5^{\text {th }}$ metrics with a down arrow $\downarrow$ is called positive metrics, implying just the opposite meaning.

From Equation (4) we can see the following: 1) the "Aiming" defined by the $1^{\text {st }}$ sub-equation is for checking the rate or percentage of the correctly predicted labels over the practically predicted labels; 2 ) the "Coverage" defined in the $2^{\text {nd }}$ sub-equation is for checking the rate of the correctly predicted labels over the actual labels in the system concerned; 3 ) the "Accuracy" in the $3^{\text {rd }}$ sub-equation is for checking the average ratio of correctly predicted labels over the total labels including correctly and incorrectly predicted labels as well as those real labels but are missed in the prediction; 4) the "Absolute true" in the $4^{\text {th }}$ sub-equation is for checking the ratio of the perfectly or completely correct prediction events over the total prediction events; 5) the "Absolute false" in the $5^{\text {th }}$ sub-equation is for checking the ratio of the completely wrong prediction over the total prediction events.

\subsection{Comparison with the State-of-the-Art Predictor}

Listed in Table 1 are the rates achieved by the current pLoc_Deep-mGpos predictor via the cross validations on the same experiment-confirmed dataset as used in [4]. For facilitating comparison, listed there are also the corresponding results obtained by the pLoc_bal-mGpos [4], the existing most powerful predictor for identifying the subcellular localization of Gram positive proteins with both single and multiple location sites. As shown in Table 1, the newly proposed predictor pLoc_Deep-mGpos is remarkably superior to the existing state-of-the-art predictor pLoc_bal-mGpos in all the five metrics. Particularly, it can be seen from the table that the absolute true rate achieved by the new predictor is over $99 \%$, which is far beyond the reach of any other existing methods [43-48]. This is because it is extremely difficult to enhance the absolute true rate of a prediction method for a multi-label system as clearly elucidated in [4]. Actually, to avoid embarrassment, many investigators even chose not to mention the metrics of absolute true rate in dealing with multi-label systems (see, e.g., [49-51]).

Moreover, to in-depth examine the prediction quality of the new predictor for the proteins in each of the subcellular locations concerned (cf. Table 2), we used a set of four intuitive metrics that were derived in [52] based on the Chou's symbols introduced for studying protein signal peptides [53] and that have ever since been widely concurred or justified (see, e.g., [52, 54-57]. For the current study, the set of metrics can be formulated as:

Table 2. Performance of pLoc_Deep-mGpos for each of the 4 subcellular locations.

\begin{tabular}{cccccc}
\hline$i$ & Location $^{\mathrm{a}}$ & $\operatorname{Sn}(i)^{\mathrm{b}}$ & $\operatorname{Sp}(i)^{\mathrm{b}}$ & $\operatorname{Acc}(i)^{\mathrm{b}}$ & $\operatorname{MCC}(i)^{\mathrm{b}}$ \\
\hline 1 & Cell membrane & 0.9747 & 0.9937 & 0.9879 & 0.9703 \\
2 & Cell wall & 0.9214 & 0.9984 & 0.9955 & 0.9424 \\
3 & Cytoplasm & 0.9779 & 0.9865 & 0.9833 & 0.9630 \\
4 & Extracell & 0.9850 & 0.9864 & 0.9864 & 0.9694 \\
\hline
\end{tabular}

${ }^{a}$ See Equation (1) and relevant context as well as the Supporting Information S1 for further explanation. ${ }^{\mathrm{b}}$ See Equation (6) for the metrics definition. 


$$
\begin{cases}\operatorname{Sn}(i)=1-\frac{N_{-}^{+}(i)}{N^{+}(i)} & 0 \leq \mathrm{Sn} \leq 1 \\ \operatorname{Sp}(i)=1-\frac{N_{+}^{-}(i)}{N^{-}(i)} & 0 \leq \mathrm{Sp} \leq 1 \\ \operatorname{Acc}(i)=1-\frac{N_{-}^{+}(i)+N_{+}^{-}(i)}{N^{+}(i)+N^{-}(i)} & 0 \leq \mathrm{Acc} \leq 1 \\ \operatorname{MCC}(i)=\frac{1-\left(\frac{N_{-}^{+}(i)}{N^{+}(i)}+\frac{N_{+}^{-}(i)}{N^{-}(i)}\right)}{\sqrt{\left(1+\frac{N_{+}^{-}(i)-N_{-}^{+}(i)}{N^{+}(i)}\right)\left(1+\frac{N_{-}^{+}(i)-N_{+}^{-}(i)}{N^{-}(i)}\right)}} & -1 \leq \mathrm{MCC} \leq 1 \\ (i=1,2,3,4) & \end{cases}
$$

where Sn, Sp, Acc, and MCC represent the sensitivity, specificity, accuracy, and Mathew's correlation coefficient, respectively, and $i$ denotes the $i$-th subcellular location (or subset) in the benchmark dataset. $N^{+}(i)$ is the total number of the samples investigated in the $i$-th subset, whereas $N_{-}^{+}(i)$ is the number of the samples in $N^{+}(i)$ that are incorrectly predicted to be of other locations; $N^{-}(i)$ is the total number of samples in any locations but not the $i$-th location, whereas $N_{+}^{-}(i)$ is the number of the samples in $N^{-}(i)$ that are incorrectly predicted to be of the $i$-th location.

Listed in Table 2 are the results achieved by pLoc_Deep-mGpos for the Gram positive proteins in each of 6 subcellular locations. As we can see from the table, nearly all the success rates achieved by the new predictor for the Gram positive proteins in each of the 4 subcellular locations are within the range of $92 \%-99 \%$, which is once again far beyond the reach of any of its counterparts.

Meanwhile, as a byproduct, the present paper has also stimulated some very interesting or provoked papers (see, e.g., [58-63]).

\subsection{Web Server and User Guide}

As pointed out in [64], user-friendly and publicly accessible web-servers represent the future direction for developing practically more useful predictors. Actually, user-friendly web-servers will significantly enhance the impacts of theoretical work because they can attract the broad experimental scientists [31]. In view of this, the web-server of the current pLoc_Deep-mGpos predictor has also been established at http://www.jci-bioinfo.cn/pLoc_Deep-mGpos/, by which users can easily get their desired data without the need to go thru the mathematical details.

\section{CONCLUSION}

It is anticipated that the pLoc_Deep-mGpos predictor holds very high potential to become a useful high throughput tool in identifying the subcellular localization of Gram positive proteins, particularly for finding multi-target drugs that is currently a very hot trend in drug development. Most important is that the predictor will become a very useful tool for fighting against the coronavirus to save the mankind in this planet.

\section{ACKNOWLEDGEMENTS}

This work was supported by the grants from the National Natural Science Foundation of China (No. 31560316, 61261027, 61262038, 61202313 and 31260273), the Province National Natural Science Foundation of Jiangxi (No. 20132BAB201053), the Jiangxi Provincial Foreign Scientific and Technological Cooperation Project (No. 20120BDH80023), the Department of Education of Jiangxi Province (GJJ160866). 


\section{CONFLICTS OF INTEREST}

The authors declare no conflicts of interest regarding the publication of this paper.

\section{REFERENCES}

1. Ehrlich, J.S., Hansen, M.D. and Nelson, W.J. (2002) Spatio-Temporal Regulation of Rac1 Localization and Lamellipodia Dynamics during Epithelial Cell-Cell Adhesion. Developmental Cell, 3, 259-270.

https://doi.org/10.1016/S1534-5807(02)00216-2

2. Glory, E. and Murphy, R.F. (2007) Automated Subcellular Location Determination and High-Throughput Microscopy. Developmental Cell, 12, 7-16. https://doi.org/10.1016/j.devcel.2006.12.007

3. Chou, K.C. (2015) Impacts of Bioinformatics to Medicinal Chemistry. Medicinal Chemistry, 11, 218-234. https://doi.org/10.2174/1573406411666141229162834

4. Xiao, X., Cheng, X., Chen, G., Mao, Q. and Chou, K.C. (2019 pLoc_bal-mGpos: Predict Subcellular Localization of Gram-Positive Bacterial Proteins by Quasi-Balancing Training Dataset and PseAAC. Genomics, 111, 886-892. https://doi.org/10.1016/j.ygeno.2018.05.017

5. Nakai, K. and Kanehisa, M. (1992) A Knowledge Base for Predicting Protein Localization Sites in Eukaryotic Cells. Genomics, 14, 897-911. https://doi.org/10.1016/S0888-7543(05)80111-9

6. Cedano, J., Aloy, P., Perez-Pons, J.A. and Querol, E. (1997) Relation between Amino Acid Composition and Cellular Location of Proteins. Journal of Molecular Biology, 266, 594-600.

https://doi.org/10.1006/jmbi.1996.0804

7. Reinhardt, A. and Hubbard, T. (1998) Using Neural Networks for Prediction of the Subcellular Location of Proteins. Nucleic Acids Research, 26, 2230-2236. https://doi.org/10.1093/nar/26.9.2230

8. Chou, K.C. and Shen, H.B. (2007) Recent Progresses in Protein Subcellular Location Prediction. Analytical Biochemistry, 370, 1-16. https://doi.org/10.1016/j.ab.2007.07.006

9. Chou, K.C., Wu, Z.C. and Xiao, X. (2011) iLoc-Euk: A Multi-Label Classifier for Predicting the Subcellular Localization of Singleplex and Multiplex Eukaryotic Proteins. PLoS One, 6, e18258.

https://doi.org/10.1371/journal.pone.0018258

10. Mandal, M., Mukhopadhyay, A. and Maulik, U. (2015) Prediction of Protein Subcellular Localization by Incorporating Multiobjective PSO-Based Feature Subset Selection into the General Form of Chou's PseAAC. Medical \& Biological Engineering \& Computing, 53, 331-344. https://doi.org/10.1007/s11517-014-1238-7

11. Maxwell, A., Li, R., Yang, B., Weng, H., Ou, A., Hong, H., Zhou, Z., Gong, P. and Zhang, C. (2017) Deep Learning Architectures for Multi-Label Classification of Intelligent Health Risk Prediction. BMC Bioinformatics, 18, 523. https://doi.org/10.1186/s12859-017-1898-z

12. Khan, S., Khan, M., Iqbal, N., Hussain, T., Khan, S.A. and Chou, K.C. (2019) A Two-Level Computation Model Based on Deep Learning Algorithm for Identification of piRNA and Their Functions via Chou's 5-Steps Rule. International Journal of Peptide Research and Therapeutics. https://doi.org/10.1007/s10989-019-09887-3

13. Khan, Z.U., Ali, F., Khan, I.A., Hussain, Y. and Pi, D. (2019) iRSpot-SPI: Deep Learning-Based Recombination Spots Prediction by Incorporating Secondary Sequence Information Coupled with Physio-Chemical Properties via Chou's 5-Step Rule and Pseudo Components. Chemometrics and Intelligent Laboratory Systems (CHEMOLAB), 189, 169-180. https://doi.org/10.1016/j.chemolab.2019.05.003

14. Nazari, I., Tahir, M., Tayari, H. and Chong, K.T. (2019) iN6-Methyl (5-Step): Identifying RNA N6-Methyladenosine Sites Using Deep Learning Mode via Chou's 5-Step Rules and Chou's General PseKNC. Chemometrics and Intelligent Laboratory Systems (CHEMOLAB), 193, 103811. https://doi.org/10.1016/j.chemolab.2019.103811 
15. Ehsan, A., Mahmood, M.K., Khan, Y.D., Barukab, O.M., Khan, S.A. and Chou, K.C. (2019) iHyd-PseAAC (EPSV): Identify Hydroxylation Sites in Proteins by Extracting Enhanced Position and Sequence Variant Feature via Chou's 5-Step Rule and General Pseudo Amino Acid Composition. Current Genomics, 20, 124-133. https://doi.org/10.2174/1389202920666190325162307

16. Malebary, S.J., Rehman, M.S.U. and Khan, Y.D. (2019) iCrotoK-PseAAC: Identify lysine Crotonylation Sites by Blending Position Relative Statistical Features According to the Chou's 5-Step Rule. PLoS One, 14, e0223993. https://doi.org/10.1371/journal.pone.0223993

17. Chou, K.C. (2011) Some Remarks on Protein Attribute Prediction and Pseudo Amino Acid Composition (50th Anniversary Year Review, 5-Steps Rule). Journal of Theoretical Biology, 273, 236-247.

https://doi.org/10.1016/j.jtbi.2010.12.024

18. Chou, K.C. (2001) Prediction of Protein Cellular Attributes Using Pseudo Amino Acid Composition. Proteins. Structure, Function, and Genetics (Erratum: ibid., 2001, Vol. 44, 60), 43, 246-255. https://doi.org/10.1002/prot.1035

19. Chou, K.C. (2005) Using Amphiphilic Pseudo Amino Acid Composition to Predict Enzyme Subfamily Classes. Bioinformatics, 21, 10-19. https://doi.org/10.1093/bioinformatics/bth466

20. Zhou, X.B., Chen, C., Li, Z.C. and Zou, X.Y. (2007) Using Chou's Amphiphilic Pseudo Amino Acid Composition and Support Vector Machine for Prediction of Enzyme Subfamily Classes. Journal of Theoretical Biology, 248, 546-551. https://doi.org/10.1016/j.jtbi.2007.06.001

21. Zhang, S.W., Chen, W., Yang, F. and Pan, Q. (2008) Using Chou's Pseudo Amino Acid Composition to Predict Protein Quaternary Structure: A Sequence-Segmented PseAAC Approach. Amino Acids, 35, 591-598. https://doi.org/10.1007/s00726-008-0086-x

22. Qiu, J.D., Huang, J.H., Liang, R.P. and Lu, X.Q. (2009) Prediction of G-Protein-Coupled Receptor Classes Based on the Concept of Chou's Pseudo Amino Acid Composition: An Approach from Discrete Wavelet Transform. Analytical Biochemistry, 390, 68-73. https://doi.org/10.1016/j.ab.2009.04.009

23. Mohabatkar, H. (2010) Prediction of Cyclin Proteins Using Chou's Pseudo Amino Acid Composition. Protein \& Peptide Letters, 17, 1207-1214. https://doi.org/10.2174/092986610792231564

24. Qiu, J.D., Suo, S.B., Sun, X.Y., Shi, S.P. and Liang, R.P. (2011) OligoPred: A Web-Server for Predicting Homo-Oligomeric Proteins by Incorporating Discrete Wavelet Transform into Chou's Pseudo Amino Acid Composition. Journal of Molecular Graphics \& Modelling, 30, 129-134. https://doi.org/10.1016/j.jmgm.2011.06.014

25. Nanni, L., Lumini, A., Gupta, D. and Garg, A. (2012) Identifying Bacterial Virulent Proteins by Fusing a Set of Classifiers Based on Variants of Chou's Pseudo Amino Acid Composition and on Evolutionary Information. IEEE-ACM Transaction on Computational Biolology and Bioinformatics, 9, 467-475. https://doi.org/10.1109/TCBB.2011.117

26. Khosravian, M., Faramarzi, F.K., Beigi, M.M., Behbahani, M. and Mohabatkar, H. (2013) Predicting Antibacterial Peptides by the Concept of Chou's Pseudo Amino Acid Composition and Machine Learning Methods. Protein \& Peptide Letters, 20, 180-186. https://doi.org/10.2174/092986613804725307

27. Kumar, R., Srivastava, A., Kumari, B. and Kumar, M. (2015) Prediction of Beta-Lactamase and Its Class by Chou's Pseudo Amino Acid Composition and Support Vector Machine. Journal of Theoretical Biology, 365, 96-103. https://doi.org/10.1016/j.jtbi.2014.10.008

28. Mei, J., Fu, Y. and Zhao, J. (2018) Analysis and Prediction of Ion Channel Inhibitors by Using Feature Selection and Chou's General Pseudo Amino Acid Composition. Journal of Theoretical Biology, 456, 41-48. https://doi.org/10.1016/j.jtbi.2018.07.040

29. Zhang, S., Yang, K., Lei, Y. and Song, K. (2019) iRSpot-DTS: Predict Recombination Spots by Incorporating the 
Dinucleotide-Based Spare-Cross Covariance Information into Chou's Pseudo Components. Genomics, 111, 1760-1770. https://doi.org/10.1016/j.ygeno.2018.11.031

30. Akbar, S., Rahman, A.U., Hayat, M. and Sohail, M. (2020) cACP: Classifying Anticancer Peptides Using Discriminative Intelligent Model via Chou's 5-Step Rules and General Pseudo Components. Chemometrics and Intelligent Laboratory (CHEMOLAB), 196, 103912. https://doi.org/10.1016/j.chemolab.2019.103912

31. Chou, K.C. (2017) An Unprecedented Revolution in Medicinal Chemistry Driven by the Progress of Biological Science. Current Topics in Medicinal Chemistry, 17, 2337-2358. https://doi.org/10.2174/1568026617666170414145508

32. Du, P., Wang, X., Xu, C. and Gao, Y. (2012) PseAAC-Builder: A Cross-Platform Stand-Alone Program for Generating Various Special Chou's Pseudo Amino Acid Compositions. Analytical Biochemistry, 425, 117-119. https://doi.org/10.1016/j.ab.2012.03.015

33. Cao, D.S., Xu, Q.S. and Liang, Y.Z. (2013) Propy: A Tool to Generate Various Modes of Chou's PseAAC. Bioinformatics, 29, 960-962. https://doi.org/10.1093/bioinformatics/btt072

34. Du, P., Gu, S. and Jiao, Y. (2014) PseAAC-General: Fast Building Various Modes of General Form of Chou's Pseudo Amino Acid Composition for Large-Scale Protein Datasets. International Journal of Molecular Sciences, 15, 3495-3506. https://doi.org/10.3390/ijms15033495

35. Chou, K.C. (2009) Pseudo Amino Acid Composition and Its Applications in Bioinformatics, Proteomics and System Biology. Current Proteomics, 6, 262-274. https://doi.org/10.2174/157016409789973707

36. Chen, W., Lei, T.Y., Jin, D.C., Lin, H. and Chou, K.C. (2014) PseKNC: A Flexible Web-Server for Generating Pseudo K-Tuple Nucleotide Composition. Analytical Biochemistry, 456, 53-60.

https://doi.org/10.1016/j.ab.2014.04.001

37. Chen, W., Lin, H. and Chou, K.C. (2015) Pseudo Nucleotide Composition or PseKNC: An Effective Formulation for Analyzing Genomic Sequences. Molecular BioSystems, 11, 2620-2634.

https://doi.org/10.1039/C5MB00155B

38. Cheng, X., Xiao, X. and Chou, K.C. (2018) pLoc_bal-mGneg: Predict Subcellular Localization of Gram-Negative Bacterial Proteins by Quasi-Balancing Training Dataset and General PseAAC. Journal of Theoretical Biology, 458, 92-102. https://doi.org/10.1016/j.jtbi.2018.09.005

39. Chou, K.C. (2019) Two kinds of Metrics for Computational Biology. Genomics. https://www.sciencedirect.com/science/article/pii/S0888754319304604?via\%3Dihub

40. Chou, K.C. (2013) Some Remarks on Predicting Multi-Label Attributes in Molecular Biosystems. Molecular Biosystems, 9, 1092-1100. https://doi.org/10.1039/c3mb25555g

41. Song, J., Wang, Y., Li, F., Akutsu, T., Rawlings, N.D., Webb, G.I. and Chou, K.C. (2018) iProt-Sub: A Comprehensive Package for Accurately Mapping and Predicting Protease-Specific Substrates and Cleavage Sites. Brief in Bioinformatics, 20, 638-658. https://doi.org/10.1093/bib/bby028

42. Zhang, M., Li, F., Marquez-Lago, T.T., Leier, A., Fan, C., Kwoh, C.K., Chou, K.C., Song, J. and Jia, C. (2019) Multiply: A Novel Multi-Layer Predictor for Discovering General and Specific Types of Promoters. Bioinformatics, 35, 2957-2965. https://doi.org/10.1093/bioinformatics/btz016

43. Shen, H.B. and Chou, K.C. (2007) Hum-mPLoc: An Ensemble Classifier for Large-Scale Human Protein Subcellular Location Prediction by Incorporating Samples with Multiple Sites. Biochemical and Biophysical Research Communications (BBRC), 355, 1006-1011. https://doi.org/10.1016/j.bbrc.2007.02.071

44. Chou, K.C. and Shen, H.B. (2008) Cell-PLoc: A Package of Web Servers for Predicting Subcellular Localization of Proteins in Various Organisms. Nature Protocols, 3, 153-162. https://doi.org/10.1038/nprot.2007.494

45. Shen, H.B. and Chou, K.C. (2009) A Top-Down Approach to Enhance the Power of Predicting Human Protein 
Subcellular Localization: Hum-mPLoc 2.0. Analytical Biochemistry, 394, 269-274.

https://doi.org/10.1016/j.ab.2009.07.046

46. Chou, K.C. and Shen, H.B. (2010) Cell-PLoc 2.0: An Improved Package of Web-Servers for Predicting Subcellular Localization of Proteins in Various Organisms. Natural Science, 2, 1090-1103. https://doi.org/10.4236/ns.2010.210136

47. Chou, K.C., Wu, Z.C. and Xiao, X. (2012) iLoc-Hum: Using Accumulation-Label Scale to Predict Subcellular Locations of Human Proteins with Both Single and Multiple Sites. Molecular Biosystems, 8, 629-641. https://doi.org/10.1039/C1MB05420A

48. Cheng, X., Xiao, X. and Chou, K.C. (2018) pLoc-mHum: Predict Subcellular Localization of Multi-Location Human Proteins via General PseAAC to Winnow out the Crucial GO Information. Bioinformatics, 34, 1448-1456. https://doi.org/10.1093/bioinformatics/btx711

49. Wang, X. and Li, G.Z. (2012) A Multi-Label Predictor for Identifying the Subcellular Locations of Singleplex and Multiplex Eukaryotic Proteins. PLoS One, 7, e36317. https://doi.org/10.1371/journal.pone.0036317

50. Huang, C. and Yuan, J. (2013) Using Radial Basis Function on the General Form of Chou's Pseudo Amino Acid Composition and PSSM to Predict Subcellular Locations of Proteins with Both Single and Multiple Sites. Biosystems, 113, 50-57. https://doi.org/10.1016/j.biosystems.2013.04.005

51. Pacharawongsakda, E. and Theeramunkong, T. (2013) Predict Subcellular Locations of Singleplex and Multiplex Proteins by Semi-Supervised Learning and Dimension-Reducing General Mode of Chou's PseAAC. IEEE Transactions on Nanobioscience, 12, 311-320. https://doi.org/10.1109/TNB.2013.2272014

52. Chen, W., Feng, P.M., Lin, H. and Chou, K.C. (2013) iRSpot-PseDNC: Identify Recombination Spots with Pseudo Dinucleotide Composition. Nucleic Acids Research, 41, e68. https://doi.org/10.1093/nar/gks1450

53. Chou, K.C. (2001) Using Subsite Coupling to Predict Signal Peptides. Protein Engineering, 14, 75-79. https://doi.org/10.1093/protein/14.2.75

54. Kabir, M. and Hayat, M. (2016) iRSpot-GAEnsC: Identifing Recombination Spots via Ensemble Classifier and Extending the Concept of Chou's PseAAC to Formulate DNA Samples. Molecular Genetics and Genomics, 291, 285-296. https://doi.org/10.1007/s00438-015-1108-5

55. Liu, B., Wang, S., Long, R. and Chou, K.C. (2017) iRSpot-EL: Identify Recombination Spots with an Ensemble Learning Approach. Bioinformatics, 33, 35-41. https://doi.org/10.1093/bioinformatics/btw539

56. Liu, B., Weng, F., Huang, D.S. and Chou, K.C. (2018) iRO-3wPseKNC: Identify DNA Replication Origins by Three-Window-Based PseKNC. Bioinformatics, 34, 3086-3093. https://doi.org/10.1093/bioinformatics/bty312

57. Feng, P., Yang, H., Ding, H., Lin, H., Chen, W. and Chou, K.C. (2019) iDNA6mA-PseKNC: Identifying DNA N(6)-Methyladenosine Sites by Incorporating Nucleotide Physicochemical Properties into PseKNC. Genomics, 111, 96-102. https://doi.org/10.1016/j.ygeno.2018.01.005

58. Chou, K.C. (2020) The Development of Gordon Life Science Institute: Its Driving Force and Accomplishments. Natural Science, 12, 202-217. https://doi.org/10.4236/ns.2020.124018

59. Chou, K.C. (2020) The Most Important Ethical Concerns in Science. Natural Science, 12, 35-36. https://doi.org/10.4236/ns.2020.122005

60. Chou, K.C. (2020) Other Mountain Stones Can Attack Jade: The 5-Steps Rule. Natural Science, 12, 59-64. https://doi.org/10.4236/ns.2020.123011

61. Chou, K.C. (2020) The Problem of Elsevier Series Journals Online Submission by Using Artificial Intelligence. Natural Science, 12, 37-38. https://doi.org/10.4236/ns.2020.122006

62. Chou, K.C. (2020) Proposing 5-Steps Rule Is a Notable Milestone for Studying Molecular Biology. Natural Science, 12, 74-79. https://doi.org/10.4236/ns.2020.123011 
63. Chou, K.C. (2020) Using Similarity Software to Evaluate Scientific Paper Quality Is a Big Mistake. Natural Science, 12, 42-58. https://doi.org/10.4236/ns.2020.123008

64. Chou, K.C. and Shen, H.B. (2009) Recent Advances in Developing Web-Servers for Predicting Protein Attributes. Natural Science, 1, 63-92. https://doi.org/10.4236/ns.2009.12011 